
\title{
25 Research Suare \\ Risk of Death: The Death Experience of Persons Admitted to US Nursing Homes
}

\section{John N Morris}

Hebrew Rehabilitation Center for the Aged Research and Training Institute: Hinda and Arthur Marcus Institute for Aging Research

Elizabeth P Howard ( $\nabla$ elizabeth.howard.3@bc.edu )

Northeastern University School of Nursing https://orcid.org/0000-0002-6282-7551

Sabrina Egge

Hannover Reinsurance, Hannover, Germany

Erez Schachter

Profility

Fredrick Sjostrand

Karolinska Institutet

\section{Research article}

Keywords: risk of death, death rate, death risk factors, nursing home admissions, MDS 3.0, interRAI

Posted Date: February 9th, 2021

DOl: https://doi.org/10.21203/rs.3.rs-195415/v1

License: (c) (i) This work is licensed under a Creative Commons Attribution 4.0 International License.

Read Full License 


\section{Abstract}

Background: An increase in both the complexity of nursing home case-mix and corresponding challenges faced by staff in planning for the care of these individuals are notable outcomes from the growth in residential and service alternatives. The purpose of this project was to identify, among long-term care nursing home residents, those personal characteristics that indicate one's risk of dying and then summarize these items in a Risk of Death Scale. In addition, an assessment of how possible confounding facility-level characteristics might impact on the risk of death model was completed.

Methods: The design of the study was a longitudinal analysis of a national cohort of US nursing home admissions from 2011 to 2012, followed over 2 years and a validation study using 2013 data The 20112012 sample included 1,536,842 nursing home admissions and the 2013 validation sample consisted of 769,148 admissions We based the Risk of Death Scale on resident characteristics. While it is based on death status at 12 months, we describe death rates under the model from one-month post-admission to two years post-admission.

Results: The Risk of Death Scale has seventeen graded levels. The lowest four scale categories (0-3) represent half the cohort with one-year death rates from $2 \%$ to $13.8 \%$, whereas the mean of the entire cohort is $24.1 \%$ at one year. The top nine categories represent approximately $9 \%$ and have one-year death rates ranging from $51.7 \%$ to $95.7 \%$.

Conclusions/Implications: The Risk of Death Scale includes a broad spectrum of 30 independent variables, including measures of prognosis, treatments, diagnoses, clinical status, function, cognitive status, and age.

\section{Background}

Nursing homes in the US serve an ever more complex patient population, ${ }^{1,2,3}$ in part reflecting the availability of community-based alternative service and residential modalities for physically and cognitively challenged older adults. Among these alternatives are assisted living residences, communitybased in-home care services, and hospice. ${ }^{4,5} 6$ With growth in residential and service alternatives, we have seen an increase in both the complexity of nursing home case-mix and corresponding challenges faced by staff in planning for the care of these individuals.

Considering this impaired case-mix large numbers of Americans will spend their last days of life in a nursing home ${ }^{7}$, and several investigators have provided insight into the needs and characteristics of these persons. Miller and Weissert ${ }^{8}$ found that death in a nursing home was more prevalent for persons who exhibit poor functional performance, severe illness, and a prior hospitalization. In a national nursing home study of residents with dementia, Mitchell and colleagues ${ }^{9}$ identified 12 variables that predicted death. Among those identified were advanced age, sex, length of stay, dyspnea, pressure ulcers, body mass index, weight loss, and congestive heart failure. A subsequent prospective study examined ongoing 
complications of residents as death approached. The study identified a more limited risk set of predisposing characteristics, including pneumonia, febrile episodes, and eating problems. ${ }^{10}$ Chen and colleagues assessed how residents with dementia approached death in contrast to persons with cancer or heart failure and in their work activities of daily living (ADL) changes in the last year of life was greatest for the terminal cancer group. ${ }^{11}$

Two other studies that addressed death in a nursing home are noteworthy. For Michigan nursing home and home care residents with dementia, Mitchell, Morris, Park, and Fries identified a series of risk factors. ${ }^{12}$ Included among the factors were hospice care, an assessed life expectancy of less than 6 months, an advance directive in place, pain, shortness of breath, and oxygen therapy. Cohen-Mansfield and colleagues reported on an eleven-year death follow-up for 399 long-term care residents. Here relevant death-risk factors included being male, a variety of medical diagnoses, agitated behavior, cognitive impairment, older age, ADL impairment, and high level of screaming. ${ }^{13}$

Others have examined the predictive validity of the Mortality Risk Index for Minimum Data Set (MDS) 3.0 using select samples and various time frames. ${ }^{14,15,16}$ Among the factors noted are: advanced age; being male; several diseases (congestive heart failure, heart failure, renal failure); and clinical complications (shortness of breath, weight loss, poor appetite). Finally, in a systematic review of deaths at home and in nursing homes, Costa and colleagues ${ }^{17}$ identified the following risk factors: receiving palliative care; dementia; stroke; judged to be in an end-stage condition; functional deficits; cancer; and cardiovascular disease.

The need to better integrate these risk factors remains for persons entering a nursing home, particularly within an environment that is serving increasingly more complex residents. In our work, we used a full two-year national admission sample of residents in US nursing homes, and made maximal use of the wide diversity of potential risk factors included in the US mandated MDS 3.0 assessment system. These data permit both a comprehensive review of risk factors and then the bringing together of these measures in a risk model that references death by day 365 of the nursing home stay enabling the construction of a Risk of Death Scale.

Our goal in this paper was first, to draw on and extend the literature of known risk factors, and secondly, to create a nationally based model to predict who will and will not die by the end of the twelve months post admission. We also examined how well this model applied to deaths for up to a two-year period.

Using this risk of death model, we also determined whether there were subsets of facilities for which the model was more or less applicable. In addition, due to our inability to track death status of residents discharged alive prior to day 365-largely going to home or hospital, we assessed whether those live discharges differed from those tracked over the one year based on their admission assessment information.

\section{Methods}




\section{Sample and Data}

The data set comprised long-term admissions to US nursing homes in the years 2011 and 2012. Excluded from the study cohort were persons admitted into post-acute (SNF) care beds in these same facilities. The US government uses its nursing-home bed resource to serve both long-stay residents (the subject of this research) and post-hospital rehabilitation patients (individuals excluded from this research). Most of the latter sub-group of residents will return home by the end of a month, some will reenter hospitals, and few will die during their stay.

The 2011-2012 cohort included 1,536,842 admissions; 764,002 for 2011 and 772,840 for 2012, and for each resident there is a government mandated, comprehensive MDS assessment at the time of admission. Follow-up assessments at 90-day intervals during the stay are also mandated, with an additional required assessment at discharge. Data are available for two years following admission. The separate model validation sample, based on long-term care admissions in 2013 to the same facilities, included 769,148 resident admissions and was used to validate the stability of the 12-month results reported in this paper.

The federally mandated Minimum Data Set (MDS) assessment for each resident (regardless of payment source) is thus the source of the independent and dependent measures used in here. The intake assessment is the source of the independent variables, while the follow-up assessments provide the information needed to construct a measure of resident status at 12 months, either alive or dead. The Centers for Medicare and Medicaid Services (CMS) mandate these assessments for each resident regardless of payor type $18,19,20,21$. Nurses and other clinical professionals, trained on the use of the MDS 3.0, complete these de-identified assessments. They are instructed to consult with the current versions of CMS's long-term care facility assessment instrument user manual to guide data collection and coding procedures. Prior research has established the reliability of such assessments, and consistent with this prior body of work we expect the reliability of these data to be acceptable. ${ }^{22} \mathrm{~A}$ baseline and discharge assessment is available for all persons, and for those who remained 90 or more days there are a series of quarterly MDS 3.0 assessments. If the person remained in the facility up to a given date or died at that date or earlier, we tracked their death status up to that point in time.

Within the MDS is an array of diverse individual elements. From these elements one can create several summary scales, including measures of cognition (CPS-cognitive performance scale), function (ADL), and frailty. We evaluated these items and scales in this work. Prior studies have established the reliability and validity of these scales embedded within the MDS and thus there use in this study. $23,24,25$

Of the items and scales in MDS 3.0 we used 160 independent variables. Most of these variables were evaluated as independent dichotomous measures (e.g., had or did not have pneumonia), but in 13 instances, multiple dichotomous forms of the MDS measure were evaluated (e.g., dichotomous variables for different age bands-age 60 or younger, age 70 or younger, age 80 or younger, age 90 or younger). These measures fall into the following categories: 
- Function: nine different ADLs (e.g., transfer support, walking support, bed mobility), a summary ADL measure, a summary frailty measure, and falls.

- Cognition and Communication: The summary CPS, memory, comatose, several measures related to specific aspects of cognition (e.g., attention), whether the person is understood by others, whether the person understands others, and speech clarity.

- Mood and Mental Health Status: Three mood items representing depression and anxiety, as well as measures describing wandering, hallucination, and delusion.

- Clinical Indicators: A judgment by the assessor of whether the person will die within six months, body mass indicators (including body mass index, weight loss, poor appetite, dehydration), system issues (including swallowing problems, shortness of breath), infections (including urinary tract infection, fever, pneumonia, wound infection, septicemia), skin and bone health (including pressure ulcers, venous ulcers, fractures), pain, internal bleeding, trouble sleeping, bladder incontinence, bowel incontinence..

- Disease Diagnoses: Twenty-two diseases were tested, including cancer, heart disease, atrial fibrillation, deep vein thrombosis, peripheral vascular disease, ortho-hypotension, coronary heart disease, cirrhosis, plegias, mental retardation/developmental disability status, respiratory failure, hepatitis, stroke, Alzheimer's, other dementia, aphasia, diabetes, renal insufficiency.

- Processes of Care: These measures include whether the person entered from a hospice, or is currently receiving hospice care. Other items reference chemotherapy, radiation, oxygen therapy, respiratory therapy, transfusion, vent or respirator, bilevel positive airway pressure/continuous positive airway pressure (BIPAP/CPAP), tracheotomy care, suctioning, medications consumed (antipsychotics, antidepressants, hypnotics, anti-coagulants, antibiotics), non-surgical dressings, respiratory therapy, respite care, isolation, physician exam, physician orders, ulcer care.

- Appliances: Use of cane/crutch, wheelchair, feeding tube, catheter, ostomy, parenteral nutrition, intravenous solution

- Demographics: Age, race, sex.

- Vision

The dependent death measure is based on MDS3.0 items that reference whether the person was still in the facility at the time of each assessment and if not, where the person was at that point in time. Using a sequence of assessments, we determined whether the person had died by the end of the year, was alive in the facility, or discharged alive (usually to home or a hospital) prior to the end of the year and did not return to the facility.

We recognize the absence of a definitive death status measure for residents discharged alive (and never returned) is noteworthy and will be addressed. Thus, for persons discharged alive before day 365 and did not return, we describe the cohort relative to the cohort for whom we have a status measure - alive or dead - at day 365. Our goal was to assess whether this sample differed from the sample of residents 
who remained in residency, thus representing a potential source of bias. These analyses are presented at the end of the results section of the paper.

Having created a risk of death scale, we assessed whether assigning facilities into distinct subgroups would change the efficacy of the risk of death scale for the residents. Several possible outcomes seemed possible: the import of the risk model would be lessened for residents in certain types of facilities; or while the risk of death scale itself would remain invariant across facilities, there would be significant differences in the distribution of residents across the categories of the risk of death scale. We tested this phenomenon by assigning facilities into categories based on the following aggregate measures: resident age, number of residents (size of facility), average resident functional status, average resident cognitive status, average RUGs case-mix, average case-mix adjusted staff size (overall and for RNs), average reported survey deficiencies, and thirty-three individual and one summary quality indicator for the facilities (e.g., percent of residents who fell over a 90-day period). For each quality indicator plus the functional, cognitive and case-mix measures, we used the national standard deviations to assign the facilities into four categories - from worst to best performance. For the deficiency, staffing, and overall quality measure we used the CMS generated five star ranking-going from a worst score of 1 to a best score of $5^{26}$. We scored average age from low to high, across five categories, while we assigned facility size measure from low thru high across six categories. We assessed how these measures related to death at the end of the 365-day period (based on the eta statistic). We selected a subset of facility measures (with the strongest association with death) to assess whether such characteristics translated both to differences in the distribution of cases across the risk of death model (which we expected), and differences in the mean death rates across the categories (which we did not expect).

Analysis

To build a broad-based risk of death model involved a two-tiered analysis. We first described the 12month death rates across the categories of each of the 160 independent variables and then constructed a logistic model based on the items with the highest multivariate odds ratios. We drew the independent variables from the baseline assessment, while the dependent measure (death) represented known status at the end of day 365 .

Our initial analyses identified the baseline independent variables that best predicted 365-day death status - alive or dead. Because of the enormous sample size, many variables, even those with low measures of association, had a significant relationship to the dependent measure. Thus, significance was not a usable way to sort through and highlight the most important of the variable in the risk pool. Rather, we focused on variables considered to have a "strong" association with mortality, in this instance a univariate odds ratio of 1.5 or higher. We also acknowledge that other investigators may have chosen another standard, something other than the 1.5 value we used. To look at the consequence of our standard, a dichotomous independent variable where we observed one category with a $22 \%$ death rate and the other with a $31 \%$ death rate, translates into a univariate odds ratio of about 1.50 or slightly higher. Such a difference is significant in the real world. 
The independent variables that met this criterion were next entered into a stepwise logistic regression model using the 2011-2012 cohort. We then replicated the model using the 2013 validation cohort.

Thru this approach, we minimize the risk of highly collinear items entering the model (e.g., while we considered many ADL measures, they largely capture the same explanatory variance relative to death, and few will enter the model). And second, this approach ensures that the model is stable over time-our findings are subject to validation using the 2013 cohort. Next, assuming a high-level of overlap in the model between the test and validation sample, the variables from the test model are used to create the Risk of Death scale. Here the contribution of each item was governed by its b-weight in the logistic model. The greater the b-weight the stronger the item's contribution to the risk of death model.

We then used the Risk of Death scale to describe the distribution of residents at the time they entered the nursing home. Next, we described death rates across the categories of the Risk of Death scale, with estimates at one month, three months, six months, nine months, twelve months, and twenty-four months. Concluding the paper, we address two key associative issues: first, does the Risk of Death scale remain invariant across different facility types; and second, how does the loss of residents because of live discharges affect our understanding of the Risk of Death model scale.

For the first of the two latter issues, recognizing that this is a new area of inquiry, we provisionally assumed that lower death rates would be seen in facilities that were larger, had higher staffing levels, treated less functionally impaired persons, treated younger persons, and delivered better quality of care.

We divided each measure into a subset of categories, and postulated that a positive relationship existed when the pattern of mean death rates was observed and the eta measure of association value was .03 or higher. We set the latter value at this low level in the belief that facility measures would have a weak effect on death rates in contrast to the stronger effects of the resident characteristics referenced above. For facility measures that met these two criteria, we completed a one-year survival model to confirm that the two extreme categories of the independent measure behaved differently over the twelve-month followup period. When this occurred we contrasted these subsets of facilities across the Death Risk scale. Our assumption was that there would be a wide difference in the distribution of cases across the categories of the Death Risk scale but more minimal differences in the death rates in these categories.

Our final analyses addressed the inability to determine whether residents discharged alive prior to day 365 and did not return to the facility, represent a major or minor distraction in our assessment of the Risk of Death scale. In a series of analyses we examined whether this sample differed from the sample of residents who remained in residency to reach the one-year point, or died within that time period.

We present two types of comparative analyses: one using the Death-Risk Model (presented in the body of the text), the second using an array of 30 independent baseline characteristics (presented in an appendix). The first set of analyses proceeded as follows: 
- We looked at the rate at which persons left the nursing home for other sites and then compared the Death-Risk Scale profiles of persons who left and did not leave the facility by day 365 . We looked at all discharged as well as those discharged home, those discharged to an acute hospital, and those discharged to another nursing home. Our question: How unique are these persons when assessed by their distribution across the Death Risk Scale?

- In a supplemental file attached to this article, we present a summary table of demographic and study variables. This file includes vulnerability items used and not used in the scale for those with followup data at year 1 and those lost to follow-up. Once again, are there major differences or do the data suggest a more homogeneous view of the two subgroups?

\section{Results}

Descriptive Characteristics

The cohort included a diverse array of residents. Demographically, $62 \%$ were female, $29.2 \%$ married, $40.4 \%$ widowed, $12.4 \%$ divorced, $1.5 \%$ separated, and $16.5 \%$ never married. The average age at admission was 76.4 years, $14.0 \%$ were under 60 and $17.1 \%$ were 90 years of age or older. Most residents came into the facility from a hospital $(76.0 \%)$ while $15.4 \%$ came from home and $5.7 \%$ from another nursing home.

Review of the cognitive items revealed that about three-quarters of residents had a memory problem, but only $4 \%$ were 'not understood by others' and $3 \%$ 'could not understand others.' The CPS showed $10 \%$ were free of cognitive deficits, while $19.8 \%$ had a moderate or more impaired cognitive status. ${ }^{24}$ We assessed resident functional performance based on the distributions for two summary scales - the Long Form (which summarizes seven ADLs) and the Nursing Home Frailty Scale. ${ }^{23}$ On the 28-point ADL Long Form scale, where higher score indicates more impairment, the mean value was 16.7 . This is $60 \%$ along the continuum, whereas at the more independent end of the continuum $13.1 \%$ of residents had a score from 0 to 10 . At the frail end of the scale, $11.6 \%$ of residents had a score of 23 or higher. A similar picture emerges on the 15-point Nursing Home Frailty Scale.

Death Rate and Relationship to Selected Demographic Characteristics

Within one month of admission, $2.1 \%$ of residents had died. This $2 \%$ monthly rate continued over the next year. By the end of six months $13.5 \%$ had died, by the end of the year $24.1 \%$ had died. Looking at age, we saw the lowest death rate for those who were less than $60-1.7 \%$ had died at 30 days, $9.4 \%$ at one-half year, and $14.8 \%$ at a year; or a loss to death rate of about $1 \%$ per month. Residents 90 or older were most likely to die $-2.9 \%$ had died at 30 days, $18.6 \%$ at one-half year, and $33.5 \%$ at one year; or about a $3 \%$ per month loss to death. Among the other demographic characteristics, we found that males were somewhat more likely to die than females $-27.5 \%$ vs. $22.1 \%$ at one year, or about a $24 \%$ difference in loss of life. The progression by race starts with an annual rate of about $17.7 \%$ for blacks and Hispanics, $19.6 \%$ for Alaskan natives, $20.6 \%$ for Hawaiian and Pacific islanders, $23.4 \%$ for Asian, and a high of $25.5 \%$ for whites. 
Death Rate and Construction of the Logistic Prediction Model

Table 1 displays the independent variables for which we observed a one-year, univariate death odds ratio of 1.50 or higher. Ninety-eight of the 160 independent variables met this criterion - or $61 \%$ of the measures. Thus, a wide variety of factors are available for the subsequent analyses. With an instrument such as the MDS 3.0 we can weave our way through an associative maze of risk factors. 
Table 1

Independent Variables Examined

\begin{tabular}{|c|c|c|c|c|c|}
\hline $\begin{array}{l}\text { DOMAIN and } \\
\text { Independent Variables }\end{array}$ & $\begin{array}{l}\% \text { in Cat } \\
\text { specified }\end{array}$ & $\begin{array}{l}\% \text { Dead at } \\
\text { One Year Cat } \\
\text { - other }\end{array}$ & $\begin{array}{l}\text { \% Dead at One } \\
\text { Year Cat - } \\
\text { specified }\end{array}$ & $\begin{array}{l}\text { Odds } \\
\text { Ratio Test } \\
\text { Sample }\end{array}$ & $\begin{array}{l}\text { Odds Ratio } \\
\text { Validation } \\
\text { Sample }\end{array}$ \\
\hline FUNCTION & 23.1 & 20.4 & 37.3 & 2.33 & 2.33 \\
\hline \multicolumn{6}{|l|}{ ADL Long 21+ } \\
\hline $\begin{array}{l}\text { Frail - Score off } 10 \text { or } \\
\text { higher on NH Frailty } \\
\text { Scale }\end{array}$ & 3.6 & 22.8 & 60.4 & 5.16 & 5.17 \\
\hline $\begin{array}{l}\text { Bathing - Limited } \\
\text { assistance + }\end{array}$ & 94.4 & 11.8 & 24.9 & 2.47 & 2.36 \\
\hline $\begin{array}{l}\text { Bathing - Extensive } \\
\text { assistance }+\end{array}$ & 88.3 & 13.8 & 25.5 & 2.14 & 2.01 \\
\hline $\begin{array}{l}\text { Bathing-- Total } \\
\text { dependence }\end{array}$ & 29.9 & 20.7 & 32.3 & 1.83 & 1.75 \\
\hline $\begin{array}{l}\text { Personal hygiene -- } \\
\text { Total dependence }\end{array}$ & 12.4 & 22.3 & 37.6 & 2.10 & 2.07 \\
\hline $\begin{array}{l}\text { Dressing }- \text { Limited } \\
\text { assistance }+\end{array}$ & 90.1 & 12.9 & 25.4 & 2.30 & 2.26 \\
\hline $\begin{array}{l}\text { Dressing-Extensive } \\
\text { assistance }+\end{array}$ & 71.9 & 16.3 & 27.2 & 1.92 & 1.89 \\
\hline $\begin{array}{l}\text { Dressing - Total } \\
\text { dependence }\end{array}$ & 11.0 & 22.5 & 37.4 & 2.06 & 2.01 \\
\hline $\begin{array}{l}\text { Locomotion on unit - } \\
\text { Limited assistance + }\end{array}$ & 77.6 & 14.9 & 26.8 & 2.10 & 2.09 \\
\hline $\begin{array}{l}\text { Locomotion on unit - } \\
\text { Extensive assistance + }\end{array}$ & 60.3 & 17.0 & 28.9 & 1.98 & 1.94 \\
\hline $\begin{array}{l}\text { Locomotion on unit - } \\
\text { Total dependence }\end{array}$ & 24.9 & 20.7 & 34.5 & 2.02 & 2.00 \\
\hline $\begin{array}{l}\text { Locomotion off unit - } \\
\text { Limited assistance + }\end{array}$ & 81.2 & 14.2 & 26.5 & 2.18 & 2.16 \\
\hline $\begin{array}{l}\text { Locomotion off unit - } \\
\text { Extensive assistance }+\end{array}$ & 67.1 & 16.3 & 28.0 & 2.00 & 1.97 \\
\hline $\begin{array}{l}\text { Locomotion off unit - } \\
\text { Total dependence }\end{array}$ & 35.9 & 19.8 & 31.9 & 1.90 & 1.85 \\
\hline $\begin{array}{l}\text { Walk in room -- Limited } \\
\text { assistance + }\end{array}$ & 82.3 & 14.9 & 26.2 & 2.02 & 2.01 \\
\hline
\end{tabular}




\begin{tabular}{|c|c|c|c|c|c|}
\hline $\begin{array}{l}\text { DOMAIN and } \\
\text { Independent Variables }\end{array}$ & $\begin{array}{l}\% \text { in Cat } \\
\text { specified }\end{array}$ & $\begin{array}{l}\text { \% Dead at } \\
\text { One Year Cat } \\
\text { - other }\end{array}$ & $\begin{array}{l}\% \text { Dead at One } \\
\text { Year Cat - } \\
\text { specified }\end{array}$ & $\begin{array}{l}\text { Odds } \\
\text { Ratio Test } \\
\text { Sample }\end{array}$ & $\begin{array}{l}\text { Odds Ratio } \\
\text { Validation } \\
\text { Sample }\end{array}$ \\
\hline $\begin{array}{l}\text { Walk on corridor - } \\
\text { Limited assistance + }\end{array}$ & 83.1 & 14.5 & 26.1 & 2.08 & 2.06 \\
\hline $\begin{array}{l}\text { Transfer }- \text { Limited } \\
\text { assistance }+\end{array}$ & 85.8 & 13.9 & 25.9 & 2.16 & 2.19 \\
\hline $\begin{array}{l}\text { Transfer - Total } \\
\text { dependence }\end{array}$ & 13.0 & 22.4 & 36.0 & 1.95 & 1.95 \\
\hline $\begin{array}{l}\text { Toilet use -- Limited } \\
\text { assistance }+\end{array}$ & 88.5 & 13.0 & 25.6 & 2.30 & 2.27 \\
\hline $\begin{array}{l}\text { Toilet use -- Total } \\
\text { dependence }\end{array}$ & 14.4 & 22.3 & 35.1 & 1.89 & 1.85 \\
\hline $\begin{array}{l}\text { Eating - Limited } \\
\text { assistance + }\end{array}$ & 31.8 & 19.5 & 34.2 & 2.15 & 2.04 \\
\hline $\begin{array}{l}\text { Eating - Extensive } \\
\text { assistance + }\end{array}$ & 19.6 & 20.6 & 38.7 & 2.44 & 2.33 \\
\hline $\begin{array}{l}\text { Eating - Total } \\
\text { dependence }\end{array}$ & 7.3 & 22.9 & 40.7 & 2.32 & 2.20 \\
\hline $\begin{array}{l}\text { Bed mobility -- Limited } \\
\text { assistance + }\end{array}$ & 82.4 & 15.2 & 26.1 & 1.97 & 2.02 \\
\hline $\begin{array}{l}\text { Bed mobility - Total } \\
\text { dependence }\end{array}$ & 8.0 & 23.0 & 37.9 & 2.05 & 2.02 \\
\hline $\begin{array}{l}\text { Balance transfer -- Not } \\
\text { steady or did not do }\end{array}$ & 88.9 & 14.8 & 25.3 & 1.95 & 1.90 \\
\hline $\begin{array}{l}\text { Balance transfer -- Did } \\
\text { not do }\end{array}$ & 12.4 & 22.6 & 35.3 & 1.88 & 1.87 \\
\hline $\begin{array}{l}\text { Balance walking -- Not } \\
\text { steady or did not do }\end{array}$ & 89.0 & 15.0 & 24.2 & 1.92 & 1.81 \\
\hline $\begin{array}{l}\text { Balance turn around - } \\
\text { not steady or did not } \\
\text { do }\end{array}$ & 89.5 & 15.3 & 25.2 & 1.87 & 1.80 \\
\hline $\begin{array}{l}\text { Balance transfer toilet - } \\
\text { - not steady or did not } \\
\text { do }\end{array}$ & 89.0 & 14.9 & 25.3 & 1.93 & 1.88 \\
\hline $\begin{array}{l}\text { Balance transfer toilet - } \\
\text { - did not do }\end{array}$ & 15.6 & 22.1 & 35.1 & 1.91 & 1.88 \\
\hline Fell & 11.0 & 23.4 & 30.7 & 1.50 & 1.42 \\
\hline
\end{tabular}




\begin{tabular}{|c|c|c|c|c|c|}
\hline $\begin{array}{l}\text { DOMAIN and } \\
\text { Independent Variables }\end{array}$ & $\begin{array}{l}\% \text { in Cat } \\
\text { specified }\end{array}$ & $\begin{array}{l}\text { \% Dead at } \\
\text { One Year Cat } \\
\text { - other }\end{array}$ & $\begin{array}{l}\text { \% Dead at One } \\
\text { Year Cat - } \\
\text { specified }\end{array}$ & $\begin{array}{l}\text { Odds } \\
\text { Ratio Test } \\
\text { Sample }\end{array}$ & $\begin{array}{l}\text { Odds Ratio } \\
\text { Validation } \\
\text { Sample }\end{array}$ \\
\hline Comatose - Yes & 0.3 & 24.1 & 45.6 & 2.64 & 2.51 \\
\hline $\begin{array}{l}\text { Cognitive Performance } \\
\text { Scale (CPS) - } 4 \text { or } \\
\text { higher }\end{array}$ & 15.4 & 21.8 & 37.3 & 2.14 & 2.06 \\
\hline $\begin{array}{l}\text { Understand others -- } \\
\text { Sometimes or never }\end{array}$ & 14.3 & 22.1 & 36.7 & 2.05 & 1.98 \\
\hline $\begin{array}{l}\text { Understood others -- } \\
\text { Sometimes or never }\end{array}$ & 13.5 & 22.2 & 36.8 & 2.05 & 1.97 \\
\hline $\begin{array}{l}\text { Speech clarity -- Not } \\
\text { clear }\end{array}$ & 12.7 & 22.6 & 34.7 & 1.82 & 1.78 \\
\hline $\begin{array}{l}\text { Inattention focusing -- } \\
\text { Yes }\end{array}$ & 17.1 & 22.3 & 33.2 & 1.74 & 1.64 \\
\hline $\begin{array}{l}\text { Altered consciousness } \\
\text { - Yes }\end{array}$ & 4.7 & 22.8 & 51.1 & 3.53 & 3.33 \\
\hline $\begin{array}{l}\text { Psychomotor } \\
\text { retardation -Yes }\end{array}$ & 4.2 & 23.3 & 44.1 & 2.60 & 2.50 \\
\hline $\begin{array}{l}\text { Acute onset mental } \\
\text { status change - Yes }\end{array}$ & 2.4 & 23.7 & 41.8 & 2.30 & 2.15 \\
\hline \multicolumn{6}{|l|}{$\begin{array}{l}\text { MOOD AND MENTAL } \\
\text { HEALTH }\end{array}$} \\
\hline $\begin{array}{l}\text { Physical aggression -- } \\
\text { Yes }\end{array}$ & 4.5 & 23.6 & 35.3 & 1.77 & 1.71 \\
\hline $\begin{array}{l}\text { Others at risk physical } \\
\text { injury - Yes }\end{array}$ & 1.9 & 24.0 & 34.8 & 1.69 & 1.64 \\
\hline $\begin{array}{l}\text { Little interest/pleasure } \\
- \text { Yes }\end{array}$ & 17.3 & 22.8 & 30.9 & 1.52 & 1.52 \\
\hline \multicolumn{6}{|l|}{$\begin{array}{l}\text { CLINICAL } \\
\text { COMPLICATIONS }\end{array}$} \\
\hline $\begin{array}{l}\text { Prognosis }<6 \mathrm{mn} \text { to live } \\
- \text { Yes }\end{array}$ & 4.6 & 21.6 & 78.4 & 13.20 & 13.57 \\
\hline Cancer - Yes & 8.4 & 21.1 & 57.1 & 4.98 & 4.80 \\
\hline Cirrhosis - Yes & 0.9 & 24.0 & 42.3 & 2.32 & 2.45 \\
\hline Heart failure - Yes & 17.9 & 22.6 & 31.4 & 1.57 & 1.54 \\
\hline Weight loss $5 \mathrm{lbs}-$ Yes & 6.8 & 23.3 & 35.3 & 1.80 & 1.70 \\
\hline
\end{tabular}




\begin{tabular}{|c|c|c|c|c|c|}
\hline $\begin{array}{l}\text { DOMAIN and } \\
\text { Independent Variables }\end{array}$ & $\begin{array}{l}\% \text { in Cat } \\
\text { specified }\end{array}$ & $\begin{array}{l}\text { \% Dead at } \\
\text { One Year Cat } \\
\text { - other }\end{array}$ & $\begin{array}{l}\% \text { Dead at One } \\
\text { Year Cat - } \\
\text { specified }\end{array}$ & $\begin{array}{l}\text { Odds } \\
\text { Ratio Test } \\
\text { Sample }\end{array}$ & $\begin{array}{l}\text { Odds Ratio } \\
\text { Validation } \\
\text { Sample }\end{array}$ \\
\hline $\begin{array}{l}\text { Unplanned weight loss } \\
5 \% \text { or more - Yes }\end{array}$ & 5.8 & 23.4 & 36.6 & 1.89 & 1.79 \\
\hline $\begin{array}{l}\text { Body mass index (BMI) } \\
-19 \text { or lower }\end{array}$ & 13.4 & 22.9 & 35.7 & 1.93 & 1.83 \\
\hline $\mathrm{BMI}-15$ or lower & 1.7 & 23.8 & 45.1 & 2.63 & 2.56 \\
\hline $\mathrm{BMI}-18$ or lower & 9.0 & 22.8 & 37.8 & 2.05 & 1.94 \\
\hline $\mathrm{BMI}-20$ or lower & 18.9 & 21.8 & 34.2 & 1.86 & 1.77 \\
\hline $\mathrm{BMI}-25$ or lower & 51.8 & 19.0 & 29.0 & 1.74 & 1.66 \\
\hline $\begin{array}{l}\text { Malnutrition diagnosis } \\
- \text { Yes }\end{array}$ & 3.3 & 23.7 & 37.1 & 1.89 & 1.84 \\
\hline Poor appetite - Yes & 19.9 & 21.5 & 34.9 & 1.96 & 1.97 \\
\hline Dehydrated --Yes & 0.4 & 24.1 & 49.0 & 3.03 & 2.88 \\
\hline $\begin{array}{l}\text { Holds food in mouth } \\
\text { (not swallowing) - Yes }\end{array}$ & 1.5 & 23.9 & 39.5 & 2.08 & 2.07 \\
\hline $\begin{array}{l}\text { Dribbles food from } \\
\text { mouth - Yes }\end{array}$ & 0.8 & 24.0 & 41.7 & 2.26 & 2.19 \\
\hline $\begin{array}{l}\text { Cough / choke - during } \\
\text { meals - swallowing } \\
\text { meds }\end{array}$ & 2.6 & 23.7 & 39.4 & 2.09 & 2.00 \\
\hline Pain swallowing -- Yes & 2.5 & 23.8 & 36.9 & 1.87 & 1.87 \\
\hline $\begin{array}{l}\text { Swallowing problem -- } \\
\text { Yes }\end{array}$ & 5.9 & 23.3 & 37.9 & 2.01 & 1.97 \\
\hline Pressure ulcer -- Any & 15.4 & 22.3 & 34.3 & 1.82 & 1.87 \\
\hline $\begin{array}{l}\text { Pressure ulcer -- Stage } \\
1\end{array}$ & 5.1 & 23.7 & 33.5 & 1.63 & 1.64 \\
\hline $\begin{array}{l}\text { Pressure ulcer -- Stage } \\
2\end{array}$ & 7.4 & 23.2 & 36.1 & 1.87 & 1.89 \\
\hline $\begin{array}{l}\text { Pressure ulcer - Stage } \\
3\end{array}$ & 1.8 & 24.0 & 35.0 & 1.71 & 1.76 \\
\hline $\begin{array}{l}\text { Pressure ulcer -- Stage } \\
4\end{array}$ & 1.2 & 24.1 & 32.9 & 1.55 & 1.57 \\
\hline $\begin{array}{l}\text { Pressure ulcer -- } \\
\text { Slough }\end{array}$ & 2.6 & 23.8 & 38.4 & 2.00 & 2.03 \\
\hline
\end{tabular}




\begin{tabular}{|c|c|c|c|c|c|}
\hline $\begin{array}{l}\text { DOMAIN and } \\
\text { Independent Variables }\end{array}$ & $\begin{array}{l}\% \text { in Cat } \\
\text { specified }\end{array}$ & $\begin{array}{l}\text { \% Dead at } \\
\text { One Year Cat } \\
\text { - other }\end{array}$ & $\begin{array}{l}\text { \% Dead at One } \\
\text { Year Cat - } \\
\text { specified }\end{array}$ & $\begin{array}{l}\text { Odds } \\
\text { Ratio Test } \\
\text { Sample }\end{array}$ & $\begin{array}{l}\text { Odds Ratio } \\
\text { Validation } \\
\text { Sample }\end{array}$ \\
\hline $\begin{array}{l}\text { Pressure ulcer -- Deep } \\
\text { tissue }\end{array}$ & 1.8 & 23.9 & 37.1 & 1.87 & 1.96 \\
\hline $\begin{array}{l}\text { Urinary incontinence -- } \\
\text { Any }\end{array}$ & 66.6 & 16.7 & 27.9 & 1.94 & 1.90 \\
\hline $\begin{array}{l}\text { Urinary incontinence -- } \\
\text { Always }\end{array}$ & 24.3 & 21.0 & 34.0 & 1.94 & 1.90 \\
\hline $\begin{array}{l}\text { Bowel incontinence -- } \\
\text { Any }\end{array}$ & 43.0 & 18.6 & 30.4 & 1.90 & 1.82 \\
\hline $\begin{array}{l}\text { Bowel incontinence -- } \\
\text { Always }\end{array}$ & 17.1 & 21.6 & 35.0 & 1.96 & 1.91 \\
\hline Pneumonia --Yes & 6.1 & 23.6 & 32.2 & 1.54 & 1.50 \\
\hline Fever--Yes & 2.1 & 23.9 & 35.6 & 1.76 & 1.70 \\
\hline Internal bleeding - Yes & 0.7 & 24.1 & 39.1 & 2.02 & 2.17 \\
\hline $\begin{array}{l}\text { Shortness of breath -- } \\
\text { Any }\end{array}$ & 15.9 & 22.1 & 35.3 & 1.92 & 1.90 \\
\hline $\begin{array}{l}\text { Shortness of breath -- } \\
\text { Exertion }\end{array}$ & 12.7 & 22.6 & 34.9 & 1.84 & 1.86 \\
\hline $\begin{array}{l}\text { Shortness of breath -- } \\
\text { Sitting (at rest) }\end{array}$ & 4.6 & 23.1 & 45.0 & 2.72 & 2.69 \\
\hline $\begin{array}{l}\text { Shortness of breath -- } \\
\text { Lying flat }\end{array}$ & 7.9 & 22.9 & 38.6 & 2.11 & 2.05 \\
\hline \multicolumn{6}{|l|}{$\begin{array}{l}\text { PROCESSES OF CARE } \\
\text { / TREATMENTS }\end{array}$} \\
\hline $\begin{array}{l}\text { Admitted from hospice } \\
\text { - Yes }\end{array}$ & 0.7 & 23.8 & 76.7 & 10.57 & 9.87 \\
\hline In hospice care - Yes & 6.4 & 20.4 & 79.6 & 15.18 & 14.81 \\
\hline Chemotherapy - Yes & 0.4 & 24.1 & 43.8 & 2.46 & 2.30 \\
\hline Radiation -- Yes & 0.3 & 24.0 & 69.9 & 7.33 & 6.89 \\
\hline Oxygen therapy - Yes & 18.4 & 21.0 & 38.1 & 2.32 & 2.26 \\
\hline Suctioning - Yes & 1.5 & 24.0 & 34.9 & 1.70 & 1.55 \\
\hline Transfusion - Yes & 0.2 & 24.1 & 34.7 & 1.67 & 1.95 \\
\hline Ulcer care - Yes & 14.5 & 22.5 & 33.5 & 1.76 & 1.85 \\
\hline
\end{tabular}




\begin{tabular}{|c|c|c|c|c|c|}
\hline $\begin{array}{l}\text { DOMAIN and } \\
\text { Independent Variables }\end{array}$ & $\begin{array}{l}\% \text { in Cat } \\
\text { specified }\end{array}$ & $\begin{array}{l}\text { \% Dead at } \\
\text { One Year Cat } \\
\text { - other }\end{array}$ & $\begin{array}{l}\text { \% Dead at One } \\
\text { Year Cat - } \\
\text { specified }\end{array}$ & $\begin{array}{l}\text { Odds } \\
\text { Ratio Test } \\
\text { Sample }\end{array}$ & $\begin{array}{l}\text { Odds Ratio } \\
\text { Validation } \\
\text { Sample }\end{array}$ \\
\hline Age -60 or over & 89.7 & 14.8 & 25.2 & 1.94 & 1.75 \\
\hline Age -70 or over & 77.3 & 16.3 & 26.5 & 1.85 & 1.69 \\
\hline Age -80 or over & 57.4 & 18.3 & 28.5 & 1.78 & 1.65 \\
\hline Age -90 or over & 20.2 & 21.7 & 28.2 & 1.83 & 1.71 \\
\hline $\begin{array}{l}\text { Not black or Hispanic } \\
\text { (e.g., white, Asian) }\end{array}$ & 83.3 & 17.7 & 25.3 & 1.58 & \\
\hline \multicolumn{6}{|l|}{$\begin{array}{l}\text { APPLIANCES / } \\
\text { DEVICES }\end{array}$} \\
\hline Cane / crutch & 96.8 & 15.1 & 24.5 & 1.83 & 1.84 \\
\hline Feeding tube -- Yes & 1.9 & 24.0 & 34.5 & 1.67 & 1.65 \\
\hline $\begin{array}{l}\text { Indwelling catheter - } \\
\text { Yes }\end{array}$ & 10.4 & 22.7 & 37.0 & 2.01 & 2.05 \\
\hline Parenteral / IV feeding & 1.2 & 24.0 & 34.8 & 1.69 & 1.88 \\
\hline \multicolumn{6}{|l|}{ VISION } \\
\hline $\begin{array}{l}\text { Vision - Moderately } \\
\text { impaired + }\end{array}$ & 9.5 & 23.2 & 33.0 & 1.63 & 1.58 \\
\hline
\end{tabular}

The variables in Table 1 are ordered by domain. Each independent variable is presented as a 0/1 dichotomy, with category " 1 " representing the specified condition (e.g., those who are dehydrated). The statistics presented for each variable are: the percent of residents with the specified condition; the 12month percent who died for the two categories; the univariate odds ratio for the test sample and the corresponding univariate odds ratio for the validation sample. Note, in every instance the odds ratio in the validation sample is approximately the same as that observed for the test sample. Of the 98 measures, eight have a univariate odds ratio of 3.0 or higher-or $8 \%$ of the total variable pool. Ordered from highest odds ratio, these variables follow:

- The first three measures (In hospice care, Admitted from hospice, Prognosis $<6 \mathrm{mn}$ to live) reflect either a clinical judgment regarding resident status or a service decision reflecting staff expectation of the likelihood the person will die. Two of these measures represent the assignment of the person to a hospice program - which implies that extraordinary care to extend life will cease, comfort care implemented, and the person's life expectancy is less than six months. Of those admitted to the nursing home from a hospice - representing less than $1 \%$ of all residents, $76.7 \%$ died by the end of the year. Of those receiving hospice care in the nursing home at the time of the admission assessment, representing $6.4 \%$ of residents, $79.6 \%$ died by the end of the year. The third measure in this set is an assessor judgment that the person had six or fewer months to live, and it too behaved 
similarly to the two hospice measures. This judgment applied to $4.6 \%$ of residents and by the end of the year, $78.4 \%$ had died - thus aligning with the 13.2 univariate odds ratio in the test cohort and 13.57 odds ratio in the validation cohort.

- The resident issues captured by the remaining five higher odds ratio variables all translated into oneyear death rates of $50 \%$ or higher (Radiation, Score of 10 or higher NH Frailty Scale, Altered Consciousness, Cancer, Dehydrated). First, for radiation treatment, which applied to only $0.3 \%$ of residents, about $70 \%$ died by the end of the year. Next, persons judged to have a high level of impairment as assessed by the NH Frailty Scale. Such persons had a score of 10 or higher, and represented about $4 \%$ of the cohort. By the end of the year about $60 \%$ died. The three remaining variables reflected persons with either cognitive difficulties - persons with an altered consciousness who were lethargic or stuporous, or persons with discrete health problems including either cancer or dehydration.

Below are interpretations of the primary forces behind the results within each domain.

- Function. Here the interpretation is twofold. First, all of the ADLs, whether measured with respect to a diverse population in need of support or measured based on a more focused lens relative to those most in need, higher death rates are exhibited. Second, the measures of balance are all related to higher death rates and those who fall have a higher likelihood of death.

- Cognition and Communication. Severe deficits in cognitive performance - whether measured by comatose or a higher score on the CPS scale, and the three measures of communication deficits, all translate into a higher loss of life by the end of the year. In addition, three other cognitive measures on the MDS 3.0 also translated into a higher loss of life. These measures reference whether the problem is present (fluctuating or not fluctuating), and they include inattention (difficulty focusing attention), psychomotor retardation (e.g., sluggishness, staring into space), and evidence of an acute change in mental status.

- Mood and Mental Health. Three measures emerged from this domain. Two reference physical aggression - which also is correlated with poor cognitive status, while the third references a loss of interest.

- Clinical complications. A total of 37 measures, including three discussed above, were related to higher loss of life. Several are individual indicators, including cirrhosis, heart failure, pneumonia, fever, and internal bleeding. Others are items within a set of similar measures in the MDS 3.0, including those related to nutritional status, skin condition, incontinence, and shortness of breath.

- Processes of Care / Treatments. We referenced three of the variables from this domain abovehospice care and radiation. Of the remaining five measures, the two with the highest loss of life (with rates at around $40 \%$ for the year) are chemotherapy and oxygen therapy.

- Demographics. Four of the five measures in this domain reference age-a series of dichotomous cuts of the age band. 
- Appliances / Devices. Two of the four measures reference nutritional status-feeding tube, parenteral/IV feeding. One references mobility-cane/crutch. The last references continenceindwelling catheter.

- Vision. Being moderately or more impaired in vision was related to higher loss of life.

Table 2 displays the thirty variables with the highest odds ratios that entered the logistic regression model. We display the multi-variate odds ratios from the test and validation samples, and the b-weights for the test sample. We use the latter in our construction of the Risk of Death Scale.

\section{Table 2. Risk of Death Model Based on a Cross Walk of the Sum of the Variables Identified in the two subscales}

- Columns = "Very High Risk Variables;" rows = "High Risk Variables"

- Numbers in cells indicate assigned value on Risk of Death Model, from low to high Further details in Figure. 1.

- Note - there is an age adjustment to the Risk of Death scale. If the person is less than 60 years of age and the Risk of Death scale score is greater than " 6 " a value of one is added to the Risk of Death Scale (this accounts for the fact that such persons are less likely to have the conditions included in the remaining variable subscale).

\begin{tabular}{|lllll|}
\hline Number of variables & 0 Very High Risk Variables & 1 & 2 & $3+$ \\
\hline 0 High Risk Variables & 0 & 4 & 9 & 10 \\
\hline 1 & 0 & 4 & 9 & 10 \\
\hline 2 & 1 & 4 & 9 & 10 \\
\hline 3 & 2 & 5 & 9 & 10 \\
\hline 4 & 3 & 6 & 9 & 10 \\
\hline 5 & 4 & 7 & 9 & 11 \\
\hline 6 & 5 & 8 & 10 & 11 \\
\hline 7 & 6 & 9 & 10 & 11 \\
\hline 8 & 6 & 9 & 10 & 11 \\
\hline 9 & 7 & 10 & 11 & 11 \\
\hline 10 & 8 & 11 & 11 & 11 \\
\hline $11+$ & 8 & 11 & 11 & 11 \\
\hline
\end{tabular}

Of the thirty variables in the model, five reference residents receiving services or having diagnoses suggestive of a more imminent risk of death - all of which have multivariate odds ratios of 2.0 or higher. 
Included are: hospice care - which applies to $6.4 \%$ of the sample and has a multivariate odds ratio of 5.30; radiation - which applies to only $0.3 \%$ of the sample and has a multivariate odds ratio of 4.72 ; a prognosis of 6 or fewer months to live - which applied to $4.6 \%$ of the sample and has a multivariate odds ratio of 2.00. Also included are two diagnoses: cancer - which applies to $8.4 \%$ of the sample and has a multivariate odds ratio of 3.8 ; and cirrhosis - reflecting $0.9 \%$ of the sample and has a multivariate odds ratio of 3.27 .

The remaining 25 variables, all with a somewhat lower odds ratio of death, fall into the following categories: treatments, diagnoses, clinical conditions, cognition and communication, function, and age. No one subset of these measures overwhelms the model. Each contributes to an increase in the overall risk of death. Working through the logistic model, starting with function, we can see the impact of weeding out the multi-collinearity effect among the variables. Of the 34 functional variables, only three entered the final model. These included locomotion on the unit, eating, and falls. The two additional treatment measures in this set, oxygen therapy and transfusion, depict seriously compromised residents.

The Clinical Complication domain has the greatest concentration of measures in the logistic model - 15 of 37 original measures, and half of all variables in the final model. The largest concentration were the five measures relative to nutritional status - unplanned weight loss, body mass index (either 18 or lower or 25 or higher), swallowing problems, or poor appetite. Two measures related to shortness of breath and two to pressure ulcers. The remaining items were isolated measures, including heart failure, internal bleeding, and urinary incontinence.

Three risk factors suggest advanced cognitive loss. These included a moderate or more severe Cognitive Performance Scale score (equivalent to a Mini Mental Status Scale score of less than 10 ), ${ }^{24}$ altered consciousness, and difficult focusing attention during the interview.

The other items in the logistic model included physical aggression, use of an indwelling catheter, and three measures of age ( 90 or older, 80 or older, and 70 or older).

Having created the logistic model, we used the variables in Table 2 to create the Risk of Death Scale, a system for assigning persons into risk of death categories. In the summary equation, the b-weight from Table 2 are each added into a summary index when the condition for the independent variable is present. We next passed the aggregated sum of the b-weights for the risk items through a simple numeric recoding process, resulting in a final scale that ranged from 0 to 16 (see footnote below). The higher the score, the greater the likelihood of death by the end of 12 months.

Figure 1 displays the distribution of cases across the categories of the Risk of Death Scale, with estimates for both the test and validation cohorts. Notably, there is little difference between the two estimates - the model based on 2011-2012 essentially is replicated in 2013. In terms of scale distribution, most persons in the cohort are in the 5 lowest scores on the scale (66.1\%), while only $2.5 \%$ are in the 5 higher categories of the 17-point scale. In short, there is a long tail of cases with the greatest risk - the prevalence is low but their immediate risk is high. 
Figure 2 displays one-year death results across the categories of the Risk of Death Scale. The rates rise from category " 0 " at $2.3 \%$ to category " 16 " at $93 \%$. By category " 6 " one-third of the residents had died by day 365 and the rate of death exceeded $50 \%$ by category " 8 ." In line with the linear progression of rates, the eta measure of association was .460 .

Figure 3 presents the death rate profiles when we extend the follow-up window from 30 days to 2 years after admission. At 30 days post admission, only persons with Risk of Death Scale scores of 10 through 16 had a death rate of $10 \%$ or higher, rising from $12-51 \%$ with steady inter category increases. For nearly all risk categories, there is a steady increase in the percentage who die over time. For example, while categories $0-9$ on the Risk of Death Scale had death rates below $10 \%$ at 30 days, by one year only categories 0,1 , and 2 had rates at this low a level. Conversely, while only category 16 had a death rate over $50 \%$ at 30 days, by one year, categories 8 thru 16 on the Risk of Death Scale had rates of this order of magnitude. Moreover, at one-year categories 11-16 on the Risk of Death Scale had death rates at the $75 \%$ or higher level. Viewed another way, at one year only risk groups " 0 " and " 1 " with annual death rates lower than $5 \%$ could be said to exhibit rates that correspond to the average for elders in the general US population. ${ }^{27}$

Next step was to test the Risk of Death Scale for invariance across an array of facility typologies. The first step included sifting through the facility measures to identify where having more or less of a specific characteristic (e.g., facility size) translated into differing rates of death. Ten of the 48 facility characteristics met the preliminary eta criterion of .03 or higher. However, in two instances the relationship was not as expected-higher death rates accompanied higher staff levels. On the face of it this makes little sense and probably reflects an under adjustment for case-mix complexity.

Of the other measures, only three of the 34 health inspection and quality of care measures met our criterion - two relating to falls (eta $=.04$ ) and one to worsening pain (eta $=.03$ ). Of the other five, the highest eta was for the average age of residents, an eta of .070 - across the five age groupings the one year death rate ranged from $4.4-26.2 \%$. Next, the eta for facility size was .04, and here the death rate by category ranged from $27.5-20.0 \%$. Finally, facilities with a lighter functional load had an eta .035 , with death rates that ranged from $15.4-24.0 \%$.

In each instance we identified one sub-group of facilities with lower death rates and these facilities served between $3 \%$ and $6 \%$ of the residents in the study cohort. In addition, most of the measures defining these subgroups had previously been referenced in our person-based Risk of Death Scale. Included in this list are age, functional status, and falls.

Below we present a more detailed exposition for two of these facility classification measures to indicate the type of effects observed. The measures reflect the average age of residents in the facilities and the average ADL long Form score of the residents. These measures typify the pattern observed for all seven of the ten measures that met our selection criteria.

Three figures present the relevant information: 
- Figures $4 \mathrm{a}$ and $4 \mathrm{~b}$ display one-year survival curve estimates. In each instance one, of the five assessed subgroups stands out as different - having a significantly lower average death rate than the remaining subgroups - for age, this is the subgroup of facilities with the youngest residents, with age averaging less than 65; for ADL Long, this is the subgroup of facilities with an average functional score in the $0-12$ range - the more independent end of the ADL continuum.

Figures $5 \mathrm{a}$ and $5 \mathrm{~b}$ and $6 \mathrm{a}$ and $6 \mathrm{~b}$ contrast the lowest subgroup with the highest subgroup for each of the two facility defining characteristics - age and functional status. In Fig. 5a, we see the distribution of cases across the Risk of Death Scale. Here a huge difference emerged. Persons in residence in the facilities classified as Low were more likely to be in the " 0 " and " 1 " categories of the Risk of Death Scale. Yet there are persons in all risk categories, but looking at the Age variable as an example, only $4.5 \%$ of the High group were in categories 0 and 1, as contrasted with $42.8 \%$ of persons in the Low group. Extending our comparison in Figs. $6 \mathrm{a}$ and $6 \mathrm{~b}$, we see only marginal differences in the 12-month death rates when contrasting the Low and High groups across the Death Risk Scale. Thus, based on these data, the facility affect is one of a differential distribution of residents across the categories of the Risk of Death Scale and not a difference in rates of death within these categories.

The above analyses relied on cases for which we knew the residents status at day 365 of the stay - alive or dead. But some residents in the facilities had been discharged alive prior to the end of the 365-day period, and then, did not return. When this occurred we were unable to enter the residents into the timespecific death risk equation construction process. To analyze this sub-group of residents, the first step was to describe the discharge sites and time to discharge, displayed in Fig. 7. Among those discharged from within the total cohort of cases, $24.4 \%$ were discharged home to a community residence, $8.1 \%$ went to an acute hospital and, $0.7 \%$ went to another nursing home. Those discharged home returned much earlier than residents who were discharged to the other two destinations - a median of about a month and a half as compared to a median of about four months. Thus, in analyses that follow we examined differences in their Risk of Death Scale profiles.

Figures 8 and 9 display two sets of contrasts for the discharge subgroups based on their Risk of Death Scale profiles. First, for all residents discharged alive prior to day $365,22 \%$ of these persons can be found in the lowest two categories of the Risk of Death Scale, while for residents in the cohort with 12-month death status, only $10 \%$ of the subgroup are in these two categories. Thus, it is reasonable to assume that the potential (but unobserved) death rate by day 365 for the discharged cohort in its entirety would be lower than the $24.1 \%$ rate seen for residents within the 12-month death status subgroup. In Fig. 9, persons discharged alive to the community constitute the majority of cases in the 0 and 1 risk categories. Further, looking at the curves represented in the right side graph, we see that they all follow a pattern of lower representation in Death-Risk scale categories 0 and 1. Finally, the proportion of residents in the higher risk categories progresses steadily as follows: alive 365 days, discharged to nursing home, discharged to acute hospital, and known to have died by day 365. (Fig. 9) 
In a supplemental file, we present a summary table of demographic and study variables. This file includes vulnerability items not used in the scale for those with follow-up data at year 1 and those lost to followup. Among the 30 items compared, only one, age in years, had an eta value of 0.15 or higher. There were no major differences between the study sample and the group lost to follow-up for the other items.

\section{Discussion}

Based on our review of the literature and the available item set in the MDS 3.0, we evaluated a large array of items for possible inclusion in the Risk of Death Scale. Of the 160 variables assessed, ninety-eight had univariate odds ratios of 1.5 or higher, and it is this list of measures that provides us with the most comprehensive view of the factors driving death. Let us begin by reviewing the more powerful measures, followed by measures that correspond to prior research. We then follow with a listing of measures reported by others, that we could not replicate and conclude with new measures that emerged from this study.

Eight of the independent risk variables had notably higher odds ratios. These are measures that when present, translate into the highest likelihood of death in the ensuing 365-day period. Included are service measures, overall frailty, and cognitive loss.

- The service indicators are admission from a hospice program, receiving care within a hospice program while in the nursing facility, and receiving radiation.

- Other measures in this set include being assessed as having a projected life expectancy of less than 6 months, having a high Nursing Home Frailty score, and experiencing altered consciousness (being lethargic, stuporous).

The following is a list of measures we found that replicate independent variables reported in the literature. Among these measures are: functional dependency, cognitive decline, a dementia diagnosis, physical aggression, heart failure, pneumonia, fever, nutritional status, poor appetite, eating problems, weight loss, a lower body mass index, pressure ulcers, shortness of breath, oxygen therapy, and advanced age. Of measures identified by others as being relevant that we were not able to replicate, we note the following: gender, recent hospital stay, pain, and stroke. New measures identified in this work, but not generally reported in the literature, are: falls, balance, fluctuating consciousness, cirrhosis, internal bleeding, incontinence, chemotherapy, feeding tube, parenteral/IV feeding, use of a crutch or cane, indwelling catheter, and vision impairment.

The second goal in this study was to create a robust Risk of Death Scale based on resident status at the end of 365 days. This model is based on 30 variables, each weighted by its b-weight when the condition is present - thus the stronger the relationship, the greater the item's contribution to the model. Once the values were adjusted, the resulting scale went from" 0 " (no risk factors) to "16" (greatest risk). Included in the model are the following measures (with higher b-weights) that suggest the person is in more imminent danger of death: hospice care, a prognosis of 6 or fewer months to live, radiation therapy, 
cancer, and cirrhosis. The remaining 25 variables in the model are representative of all the dimensions evaluated, indicating the broad scope of the factors driving death. The variables by domain are as follows: function (locomotion on the unit, support in eating, and falls); treatments (oxygen therapy and transfusion); clinical complications (unplanned weight loss, body mass index, swallowing problem, poor appetite, shortness of breath, pressure ulcers, heart failure, internal bleeding, and urinary incontinence); cognition (more severe Cognitive Performance Score, altered consciousness, and difficult focusing attention); physical aggression; indwelling catheter; and age Additionally, analysis of 2013 data served to validate the created Risk of Death Scale.

The case distribution across the Risk of Death Scale began with $5 \%$ in category " 0 ', rising to $10-18 \%$ for categories " 2 " through " 5 ", and ending with $2 \%$ or less for categories " 8 " through " 16 ". The death rates across the scale categories progress linearly from 2-96\%. The first 3 scale categories, representing about $31 \%$ of the cohort had an average one year-death rate of less than $9 \%$. This rate is higher than the survival statistics on a general US population, where from 2011, $2.9 \%$ of women and $4.1 \%$ men aged on average as 76-years-old (age-matched to this study population) died within one year. These data correspond to the mortality rates seen for the Risk of Death Scale score of " 0 " and " 1 ". The last nine categories of the scale, representing about $9 \%$ of the cohort, had one-year death rates of $51 \%$ or higher, while we saw extreme one-year death rates of $82 \%$ or higher for the last five scale categories and representing $2.5 \%$ of the population.

When we extend death rates to 2 years post-admission, we observed a steady increase in death rates across all categories of the Risk of Death Scale. Categories " 0 " and " 1 " had the lowest death rates at all points in time - staying at about $10 \%$, even at 2 years post-admission. Category " 7 " on the Risk of Death Scale had death rates at about the population average at all follow-up time points; category " 8 " had death rates at about double the cohort average across all follow-up time periods.

In our analysis of facility characteristics that may prove to be invariant across the Death Risk model, 7 of 48 facility characteristics met our a priori criteria. These included: the falls quality indicator, the worsening pain quality indicator, average resident age, facility size, and mean functional profile of the resident. In each instance we contrasted the facilities with the lowest vs. facilities with the highest distribution of these characteristics. The effects observed were invariant across these facility classifications. There were differences in the distribution of cases across the Risk of Death Scale, but no real differences in the rate of deaths across these categories. In short, facility characteristics did not play a role in the resident death profile. The outcome is as predicted.

Conducting a secondary analysis has inherent limitations. We used an existing data set without opportunity to mandate the independent variables for inclusion. The Risk of Death Scale was designed and tested on residents in long-term care and not those receiving skilled nursing care, nor older adults in other settings. In addition, the number of cases lost to follow-up was a concern. Here our analyses, based on the Death-Risk Scale profiles, suggest that the only difference between those for whom 12-month status was known and unknown was a slightly higher percent of cases in the first two (lower) categories 
of the Risk of Death Scale - those who were less likely to die in the discharged cohort. This outcome reflects the lower risk status of those discharged home, something most likely to occur earlier in the stay. For those discharged to another nursing home or to a hospital, their risk profiles did not differ dramatically from the profile of residents for whom we knew the resident's 12-month death status, Finally, in our case analysis in the appendix, exclusive of age, these cases do not differ in their risk-profile from those for whom 1-year follow-up death data were available. Thus, they are not a distinct sub-population.

\section{Conclusions}

In this paper we demonstrated that death following admission to a nursing home is based on a diverse array of risk factors, some with very high rates, and others with more moderate rates. The summary Risk of Death Scale incorporated measures of treatments, diagnoses, death prognosis, clinical status, function, cognition, and age. The resulting Risk of Death Scale differentiates death across the categories by our earliest follow-up point (30 days) and continues to be relevant to the last of our follow-up points at 2 years. Besides the apparent clinical utility, the scale may have other uses. States in the US monitoring for excess death rates in suboptimal facilities, insurance companies providing coverage upon entry, and the basis for a length of life quality indicator are among examples of the extended utility of the Risk of Death Scale created in this effort.

\section{Abbreviations}

US United States

ADL Activities of Daily Living

MDS Minimum Data Set

SNF Skilled Nursing Facility

CMS Centers for Medicare and Medicaid Services

CPS Cognitive Performance Scale

BIPAP Bilevel positive airway pressure

CPAP Continuous positive airway pressure

RUGs Resource Utilization Groups

RN Registered Nurse

b-weight Beta weight

IV intravenous 


\section{Declarations}

Ethics and Consent to Participate

The secondary MDS 3.0 data for residents in US nursing homes were provided pursuant to an agreement with CMS in the United States. These data were anonymized before coming to the analytic team. The analyses are covered by an approval from the Hebrew Senior Life, Institute for Aging Research, Institutional Review Board, and we completed the analyses using SPSS version 20 and 22.

Availability of Data and Material

As CMS provided the data analyzed in this paper pursuant to a specific request by our research team they are not available through us for analysis by others. Others can, however, submit a request to CMS to access and analyze their MDS data holdings. CMS considers such requests on a case-by-case basis and will put restrictions on their use.

Competing Interests

JNM, EH, FS are members of interRAl.

ES is a full-time salaried employee of Profility.

FS, SE are salaried employees of Hannover Re

Disclosure

Mr. Erez Schachter has a financial interest in Profility, a company that provides software tools and services to support health care planning. The company's solutions benefit from health patterns and knowledge collected by data analysis created from patients' populations. The knowledge and patterns captured in this study are published in order for any party, including but not limited to Profility to enjoy. Erez Schachter's interests were reviewed and are managed by Hebrew SeniorLife under their conflict of interest policies."

\section{Funding}

Profility provided partial funding for this study through a contract with Hannover Re, by the Marcus Institute for Aging Research (at HebrewSenior Life), and by interRAI (an international nonprofit organization). Profility, Inc supported costs associated with planning the study design and data access.

Authors' contributions

JNM led the study design, analysis and interpretation of data, and preparation of the manuscript. EH, FS and SE provided bibliographic support for the manuscript and collaborated in project conceptualization, the interpretation of data and revision of the manuscript. The final version of the manuscript was revised and approved by all authors. 
Acknowledgements

None

\section{References}

1 Mitchell, S.L., Teno, J.M., Miller, S.C., Mor, V. (2005). A national study of the location of death for older persons with dementia. Pain and Symptom Management, 53 (2):299-305.

2 CMS. (2015). Nursing home data compendium 2015 edition. Center for Medicare and Medicaid Services, dnav.CMS.gov.

3 Sahyoun, N.R., Pratt, L.A., Leutzner, H., Dey, A., Robinson, K.N. (2001). The changing profile of nursing home residents: 1985-1997. Aging Trends; No. 4, Hyatville, Maryland, National Center for Health Statistics.

4 Grabowski, D., Stevenson, D.G., Cornell, PO.Y. (2019) Assisted living and the market for nursing home care. Health Services Research, 47(6):2296-2315.

5 Thomas, K.S., Mor, V. (2012) The relationship between older American act itle III state expenditures and prevalence of low-care nursing home resident. Health Services Research, 48(3):1215-1226.

6 Segelman, M., Intrator, O., Yue, L., Mukamel, D., Veazie, P., Temkin-Greener, H. (2017) HCBS spending and nursing home admissions for 1915-c waiver enrollees. Journal of Aging \& Social Policy, 29(5):395-412.

7 Quick stats: Percentage of deaths by place of death - national vital statistics system, United States, 2000-2018. MMWR mor6 Mortal Wkly Rep 2020; 69:611.

DOl:httpi//dxidoi.ord/10.15585/mmwr.mm691994

8 Miller, E.A. \& Weissert, G.W. (2000). Predicting elderly people's risk for nursing home placement, hospitalization, functional impairment, and mortality: a synthesis. MedicalM Care Research and Review 57, pp-pp.

9 Mitchell, S.L., Miller, S.C., Teno, J.M., Davis, R.B., Shaffer, Michele. (2010). The advanced dementia prognostic tool: a risk score to estimate survival in nursing home residents with advanced dementia. Journal of Pain and Symptom Management, 40 (5):639-652.

10 Mitchell S.L., Teno, J.M., Kiely, D.K., Shaffer, M.L., Jones, R.N., Prigerson, H.G., Volicer, L., Givens, J.L., Hamel, M.B. (2009). The clinical course of advanced dementia. New England Journal of Medicine, October 15 361:1529-1538.

11 Chen, J.H., Kiely, D.K., Morris, J.N., Mitchell, S.L. (2007). Terminal trajectories of functional decline in the long-term care setting. J Gerontol A Biol Sci Med, 62:531-536.. 
12 Mitchell, S.L., Morris, J.N., Park, P.S., Fries, B.E. (2004). Terminal care for persons with advanced dementia in the nursing home and home care settings. Palliative Care, 7 (6).

13 Cohen-Mansfield, J., Marx, M.S., Lipson, S., Merner, P. (1999). Predictors of mortality in nursing home residents. Journal of Clinical Epidemiology. 52(4):273-280.

14 Niznik, J.D., Zhang, S., Mor, M.K., Zhao, X., Ersek, M., Aspinall, S.L.,,'Thorpe, C.T. (2018). Adaptation and initial validation of minimum data set (MDS) mortality risk index to MDS version 3.0. Journal of the American Geriatrics Society, 66(12), 2353-2359.

15 Pocock, D, Parker-Oliver, D., Petroski, G.F., Rantz, M. (2010). The MDS mortality risk index: the evolution of a method for predicting 6-month mortality in nursing home residents. BMC Research Notes, 16; 3. 200.

16 Pocock, D, Parker-Oliver, D., Zweig, S., G.F., Rantz, M., Mehr, D., Madsen, R., Petroski, G., (2005).

Predicting death in the nursing home: development and validation of the 6-month minimum data set mortality index. Journal of Gerontology: Series A, Vol 60, Issye 4, April 2005, 481-498.

17 Costa, V., Earle, C.C., Esplen, M.J., Fowler, R., Goldman, R., Grossman, D., Levin, L., Manuel, D.G., Sharkey, S. Tanuseputro, P., You, J.J. The determinants of home and nursing home death: a systematic review and meta analysis. BMC Palliative Care, 15, 8 (2016).

18 Hawes, C., Morris, J.N., Phillips, C.D., Fries, B.E., Murphy, K, Mor, V. (1997). Development of the nursing home resident assessment instrument in the USA. Age and Aging, 26 (suppl 2), 19-25. https://doi.org/10.1093/aging/26.suppl_2.19.

19 Jonsson, P.V. (2005). What can we learn from the placement assessment of the elderly? Laeknabladid, The Islandic Medical Journal. 91 (2), 147-148. PMID: 16155309.

20 Rahman, A.N., Applebaum, R.A. (2009). The nursing home minimum data set assessment instrument: manifest functions and unintended consequences - past, present, and future. The Gerontologist, 49 (6), 727-735. https://doi.org/10.1093/geront/gnp066.

21 Gruber-Baldini, A.L., Zimmerman, S.I., Mortimore, E., Magaziner, J. (2000). The validity of the minimum data set in measuring the cognitive impairment of persons admitted to nursing homes. Journal of the American Geriatrics Society, 48 (12), 1601-1606. https://doi.org/10.1111/j.1532-5415.2000.tb03870.x.

22 Hirdes, J.P., Ljunggren,G., Morris, J.N., D.HM, Soveri, H. F., Gray, L., Bjorkgren, M., Gilgen, R. (2008). Reliability of the interRAl suite of assessment instruments: a 12-country study of an integrated health information system. BMC Health Services Research, 8:277.

23 Morris, J.N., Fries, B.E., Morris, S.A. (1999). Scaling adls within the MDS. Journal of Gerontology, 54(11): M546-M553. 
24 Morris, J.N., Fries, B.E., Mehr, D.R., Hawes, C., Phillips, C., Mor, V., Lipsitz, L. A. (1994). Minimum data set cognitive performance scale. Journal of Gerontology, 49(4), M174-M182 doi:

10.1093/geronj/49.4.M174

25 Howard, E., Morris, J., (2018). Frailty and recovery in the nursing home setting. Innov Aging. 2(Supple 1);717-718. Do1: 10.1093/geroni/igy023.2656.

26 Dulal, R. (2018). Technical efficiency of nursing homes: do five-star quality ratings matter? Health Care Manag Sci, 21 (3), 393-400.

27 US Social Security Administration. Actuarial Life Table, Period Life Table, 2011. SSA website. https://www.ssa.gov/oact/STATS/table4c6_2011.html. Accessed February 2, 2019.

\section{Figures}

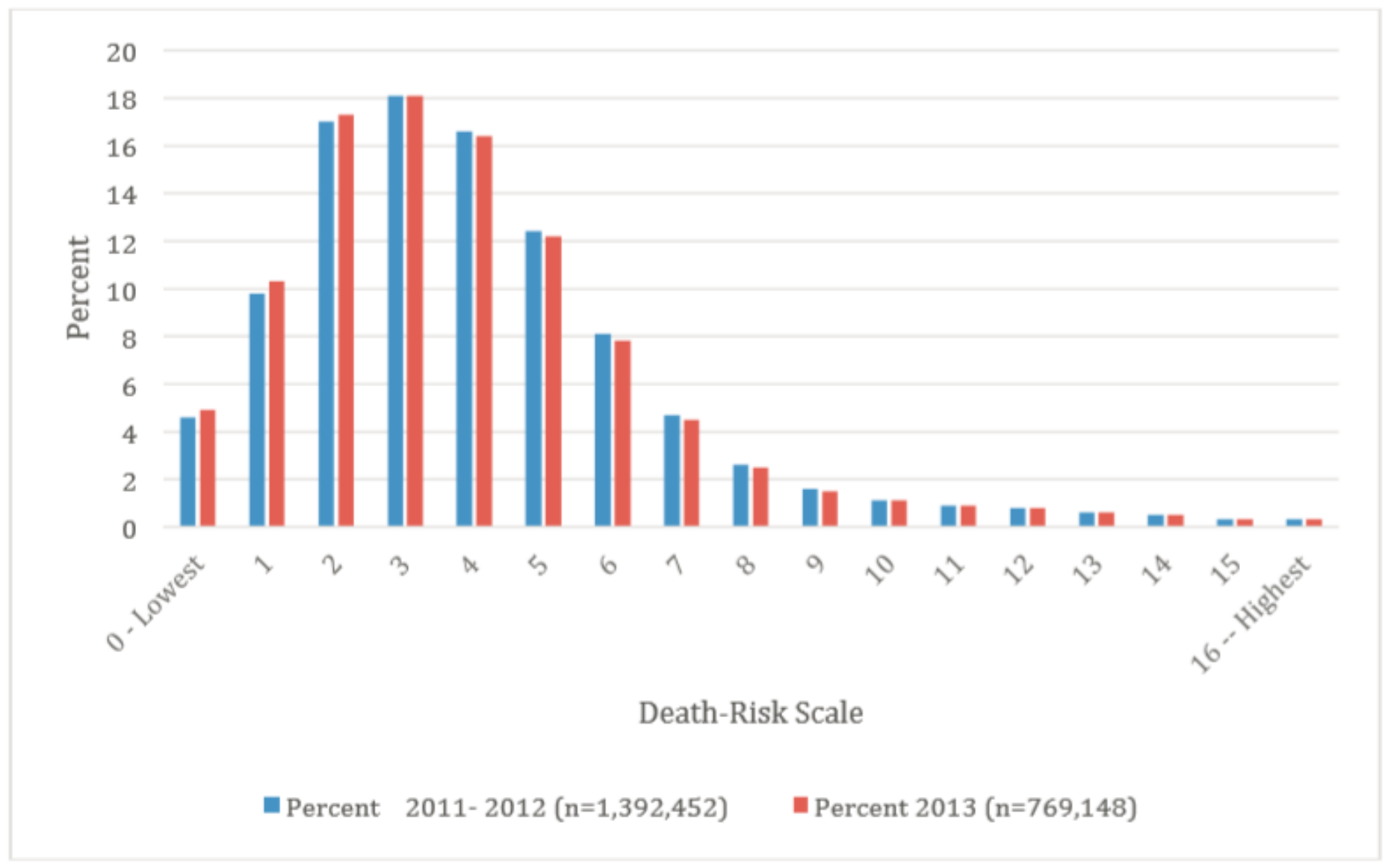

\section{Figure 1}

Risk of Death Scale Distribution in US Nursing Homes 


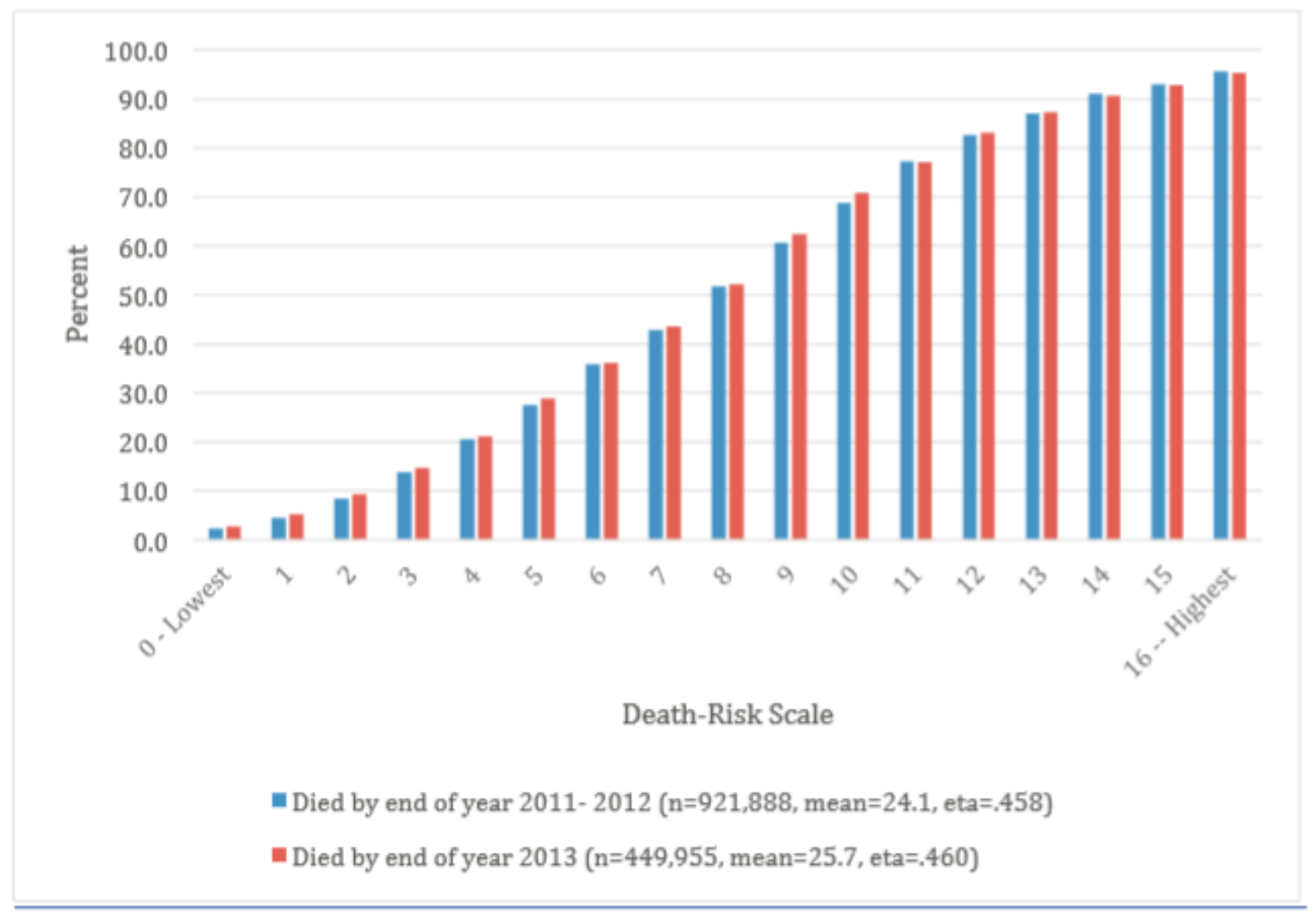

Figure 2

Percent Died by End of Year Across Categories of Death-Risk Scale 


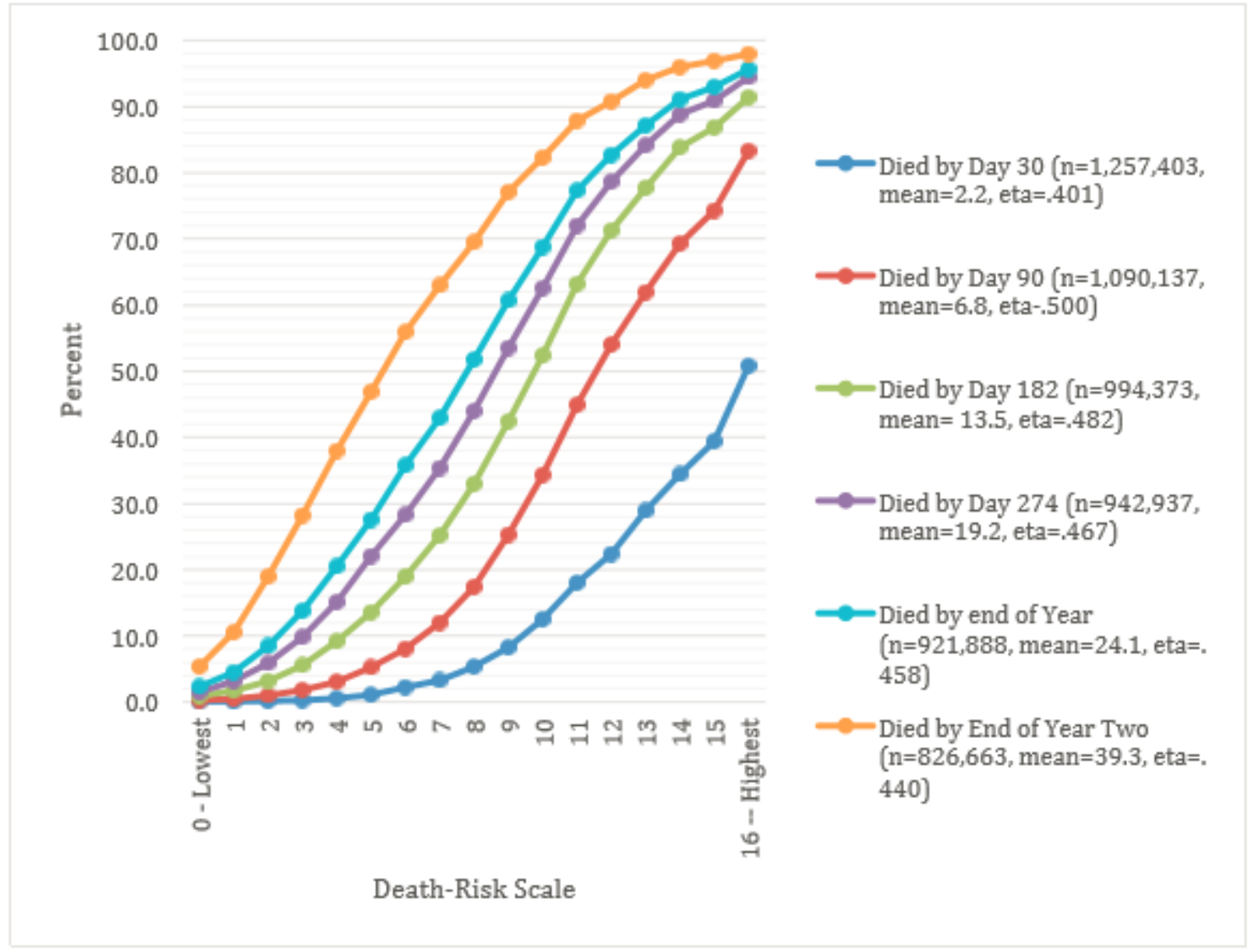

\section{Figure 3}

\section{Death Rates Over Time}
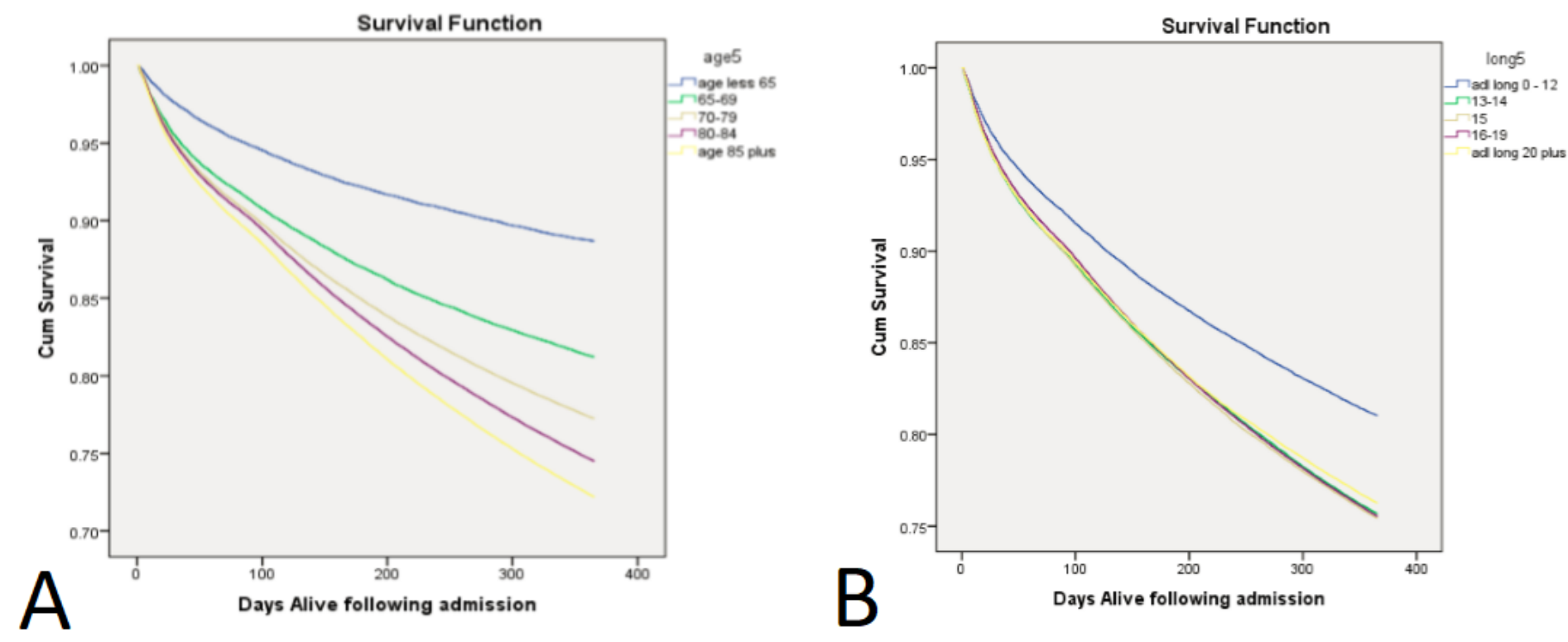

\section{Figure 4}



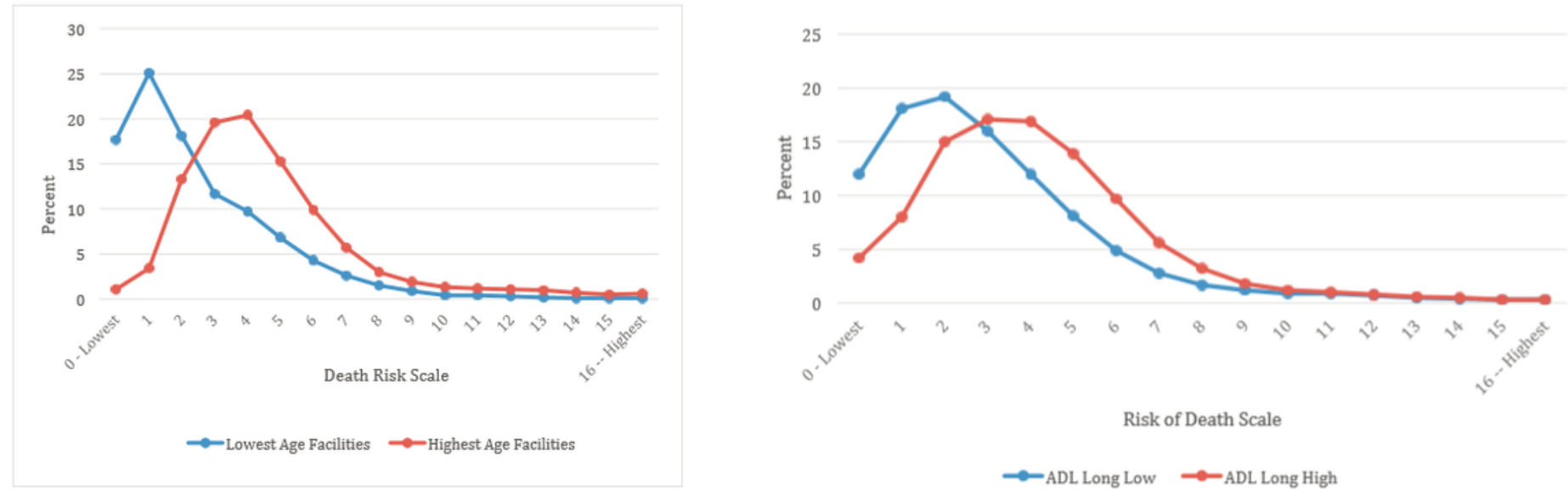

A

B

\section{Figure 5}

Distribution of Cases in Low and High Average Age Facilities Across Categories of Risk of Death Scale Distribution of Cases in High and Low ADL Long Form Facilities Across Risk of Death Scale
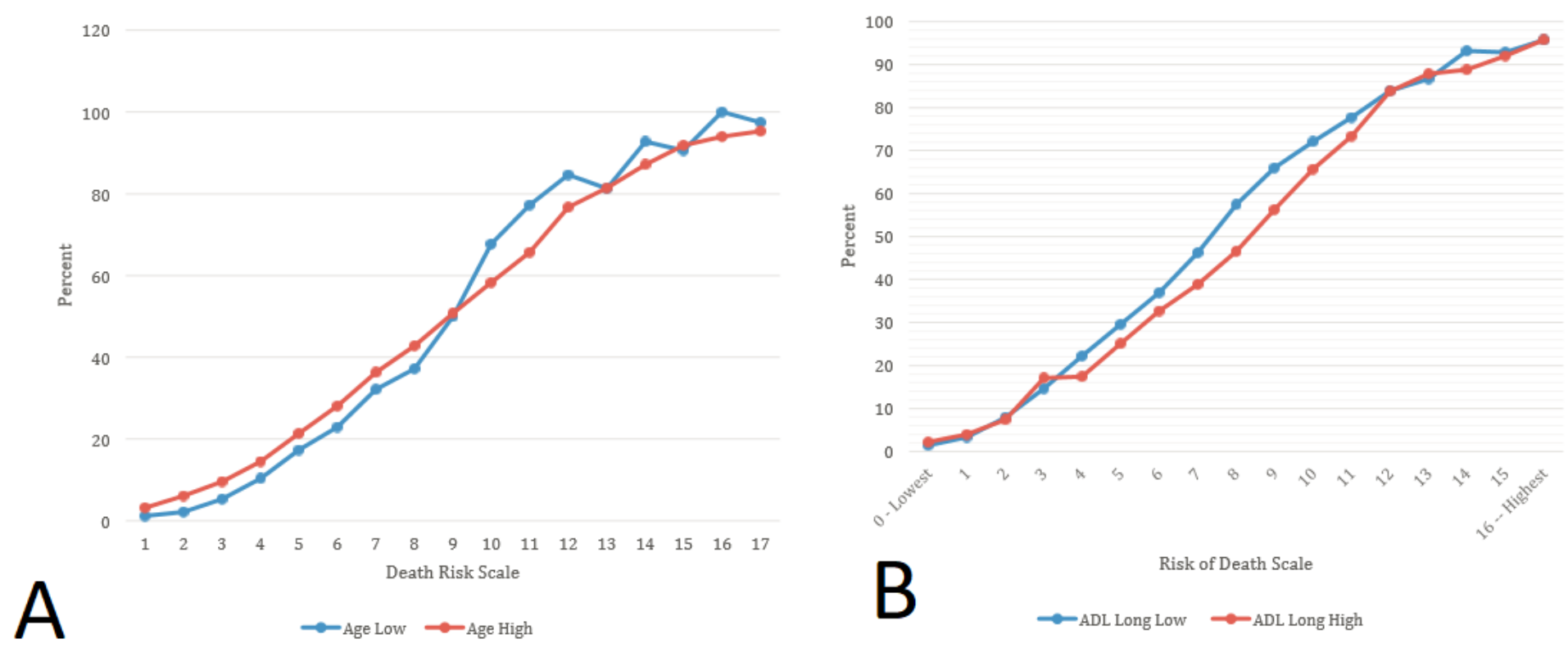

Figure 6

12-Month Rate of Death by Age Low and Age High Mix of Facilities by Risk of Death Scale 12-Month Rate of Death by Low and High ADL Mix at Facilities and Risk of Death Scale 


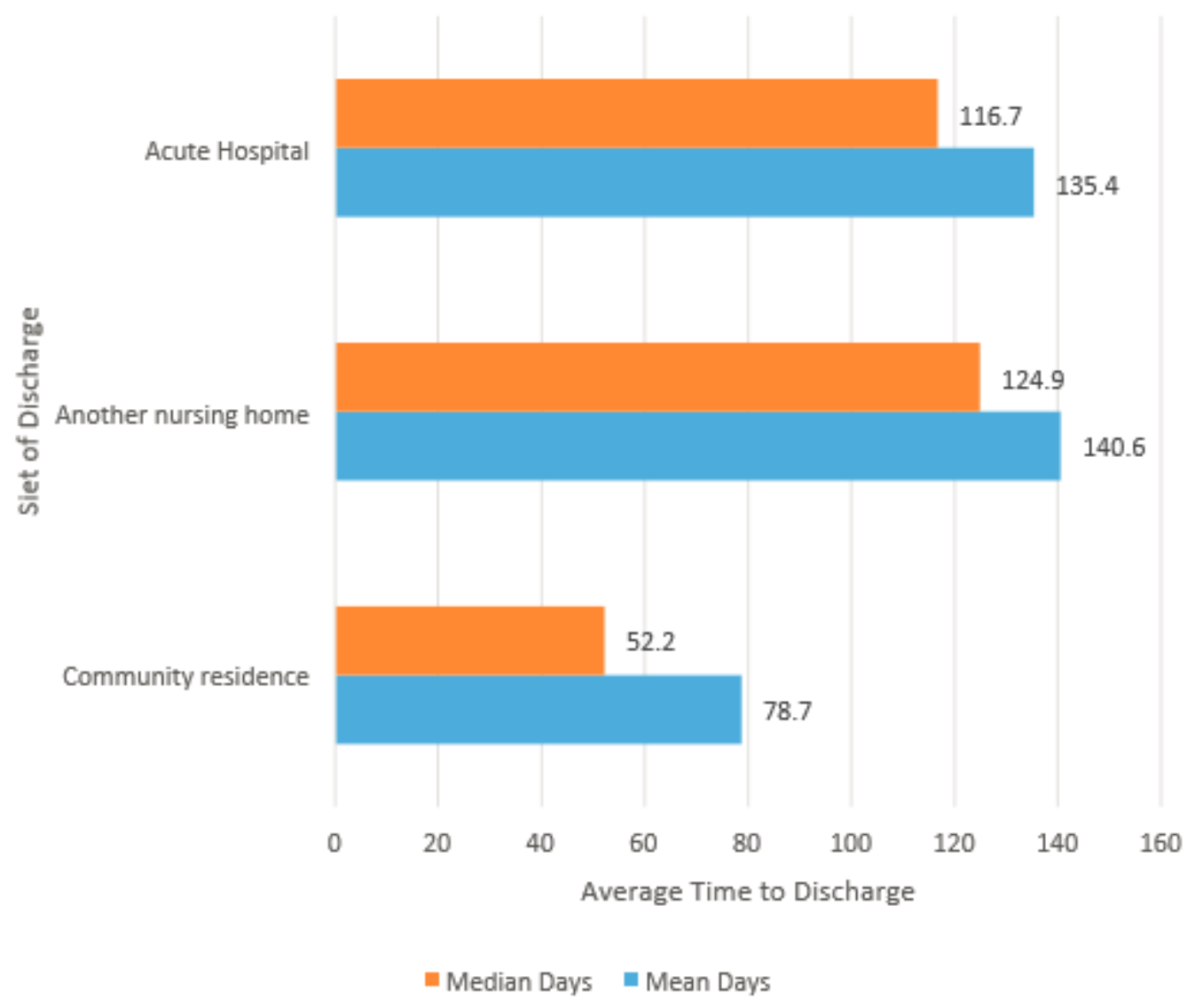

Figure 7

Average Time to Discharge and No Return
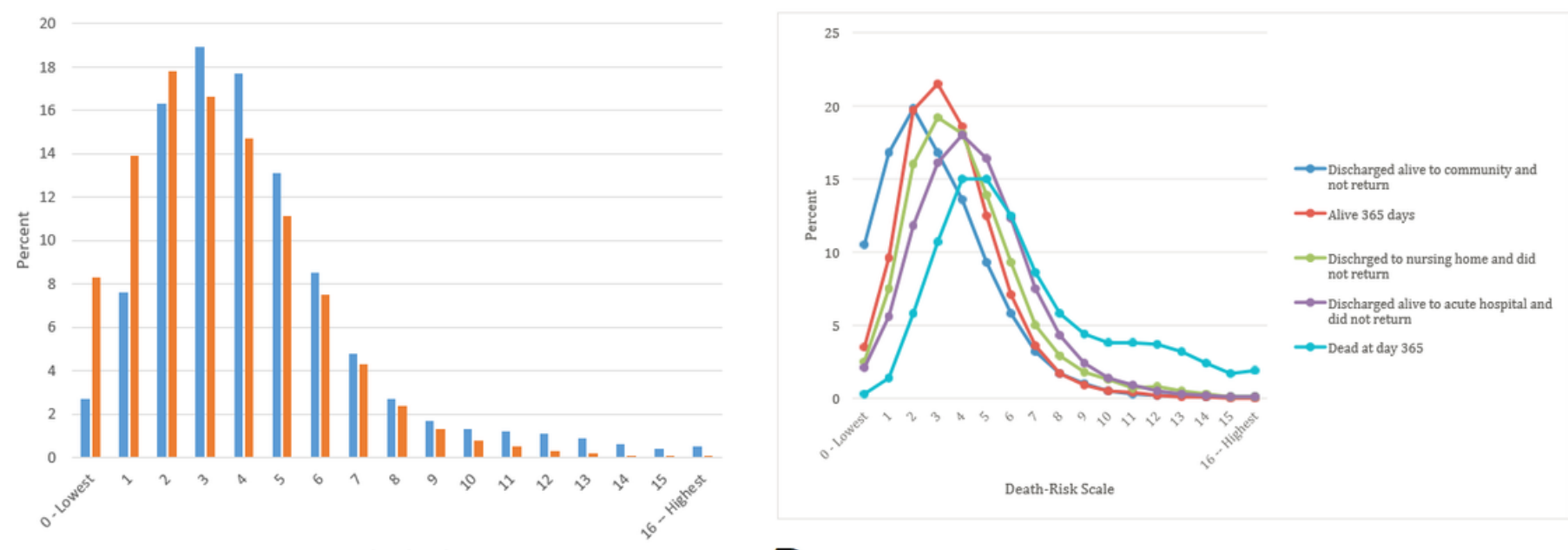

Death Risk Scale

B

\section{Figure 8}


Death Risk Scale Comparison for Persons in Death Cohort and Persons Discharged Prior to Day 365 Comparison of Death-Risk Scale Profile for Discharged and Non-Discharged Samples

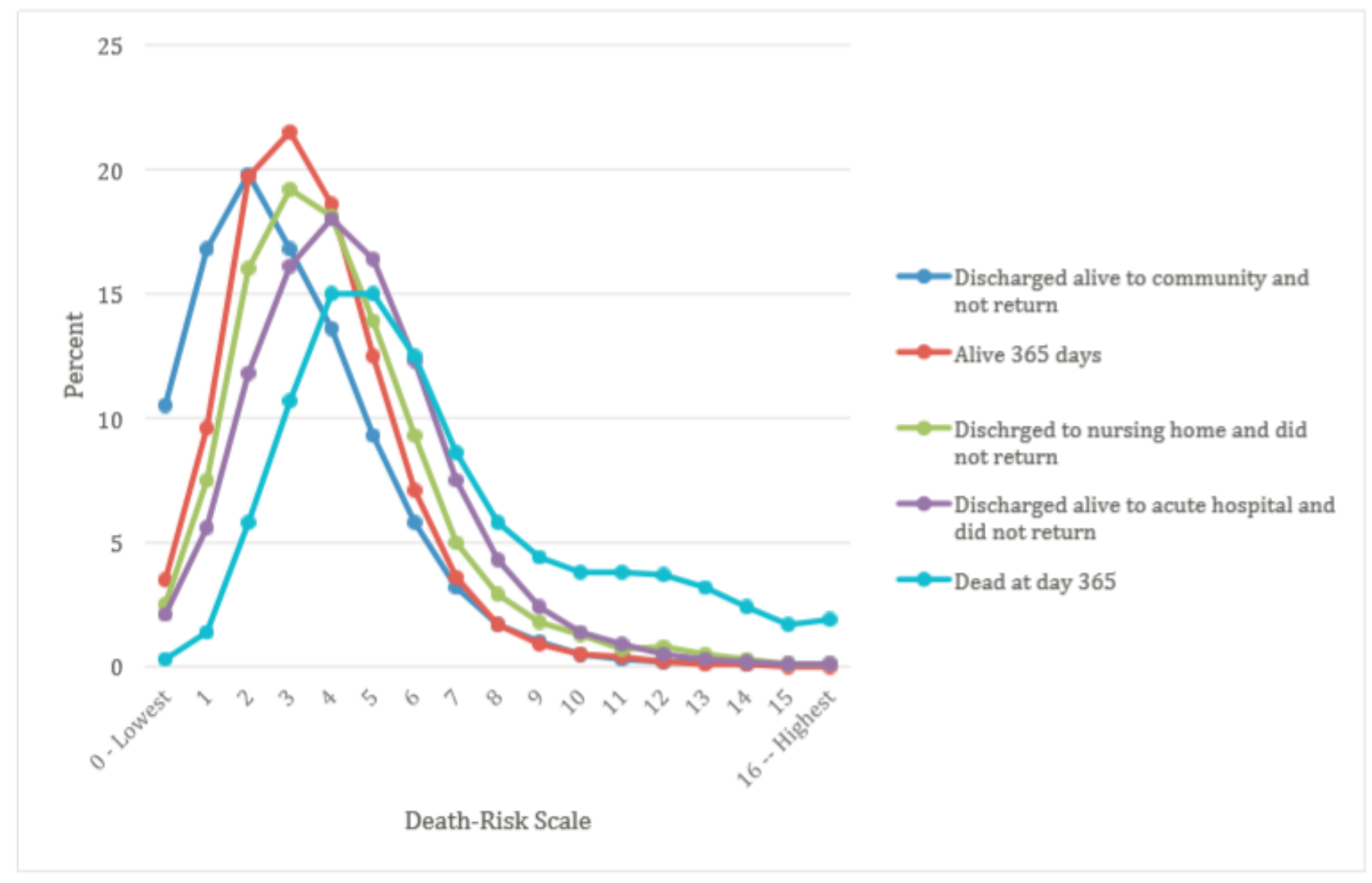

Figure 9

Comparison of Death-Risk Scale Profile for Discharged and Non-Discharged Samples

\section{Supplementary Files}

This is a list of supplementary files associated with this preprint. Click to download.

- RiskofDeathScaleappendixJan2021.docx 